\title{
Solid Target Studies for Muon Colliders and Neutrino Beams
}

\author{
N. Simos ${ }^{1 *}$, H. Kirk ${ }^{1}$, H. Ludewig ${ }^{1}$, P. Thieberger ${ }^{1}$, W-T Weng ${ }^{1}$, P.T. Trung ${ }^{1}$, K. McDonald ${ }^{2}$, J. Sheppard ${ }^{3}$, K. \\ Yoshimura $^{4}$, and Y. Hayato ${ }^{4}$ \\ ${ }^{1}$ Brookhaven National Laboratory, UPTON, NY 11973, USA \\ ${ }^{2}$ Princeton University, Princeton, NJ, USA \\ ${ }^{3}$ Stanford Linear Accelerator, Stanford, CA, USA \\ ${ }^{4}$ KEK, Tsukuba, Japan
}

This paper presents preliminary results from an ongoing post-irradiation analysis of materials that have been irradiated at the Brookhaven National Laboratory (BNL) Isotope facility. The effort is part of an experimental study that focuses on how prone to irradiation damage these materials are and thus what is their potential in playing the role of high power targets in the neutrino superbeam and the muon collider initiatives.

\section{BACKGROUND}

To address the issue of target viability and performance in the ever-increasing demand for high power accelerators, a systematic effort has been under way at BNL consisting of exposure of targets to high intensity beams [1], as well as material irradiation studies [2]. These irradiation studies focus on "smart" materials, new alloys and composites that appear to have physical and mechanical properties favorable for target survival from beam-induced shock. Since target survivability also depends on the ability of the material that it is made of to resist damage caused by the bombarding energetic particles, that information is crucial in assessing how long will a target last after it succeeds to absorb the thermal shock induced by a pulse. Relevant damage information for several materials considered for high power targets is available [3] from reactor studies and almost entirely from neutron doses. It is, however, unclear whether there is a difference between proton and neutron irradiation of materials. Irradiation damage data for the "new" and "smart" alloys and composites are far more scarce or non-existent.

In [2] the different phases of the BNL material irradiation studies were presented in detail. Specifically, in the first phase inconel-718 and super-invar where irradiated at the isotope facility receiving approximate $0.25 \mathrm{dpa}$ for the super invar. During the first post-irradiation analysis it appeared that even a small amount of irradiation was enough to dramatically alter its extremely low thermal expansion coefficient that it enjoyed in the nonirradiated state.

In the second phase of the material irradiation study, the search for new alloys, composites and "smart" materials continued and a new and expanded matrix was established for irradiation studies. It includes a 3D weaved carbon-carbon composite considered as the target candidate for the neutrino superbeam and the IG-43 graphite considered for target in the $\mathrm{T} 2 \mathrm{~K}$ neutrino beam experiment. The material assembly also includes Vascomax, Ti-6Al-4V alloy, beryllium, AlBeMet and the "smart" alloy gum metal [4] that exhibits non-elasticity, low modulus and thermal expansion as well as magnificent strength in the post-yield state (Figure 5). Lastly, specimens of nickel-plated aluminum, the same material used in the magnetic horn of the NuMi experiment was included to study the effects of irradiation on the special plating.

This paper presents preliminary results of the post-irradiation study that followed the irradiation of the assemblage of materials at the BNL isotope facility. From the wealth of results generated only a few that are especially intriguing are presented here for the lack of space. Complete results will be published in another report to follow.

\section{MATERIAL IRRADIATION STUDY}

Shown in Figure 1 is the schematic of the actual assembly of the irradiated materials. The specimens were being irradiated with the $200 \mathrm{MeV}$ beam of the BNL isotope facility that was degraded to about 104 $\mathrm{MeV}$ as the back of the last irradiation box. The approximate current associated with beam was estimated to be $70 \mu \mathrm{A}$. From radiography analysis of special nickel foils it was estimated that the beam had $\sigma_{\mathrm{x}}=8.1 \mathrm{~mm}$ and $\sigma_{\mathrm{y}}=8.4 \mathrm{~mm}$. The total proton

* Work performed under the auspices of the US DOE 
flux incident on the targets was approximately $3.7 \mathrm{x}$ $10^{20}$

An irradiation damage analysis based on damage cross sections for the materials involved using the MCNPX Monte Carlo Code [5] revealed that most of the damage, expressed in Displacements-perAtom (dpa), was from the incident protons. The contribution of neutrons to the damage was minimal. The heavier materials, such as Vascomax, Ti-6Al$4 \mathrm{~V}$ and "gum" metal, experienced irradiation damage of the order of $0.27 \mathrm{dpa}$ (protons and neutrons combined). The carbon-carbon composite and graphite received irradiation damage of 0.012 dpa. While the damage value for graphite and CC composite appear small, it has been shown that even at this level of irradiation damage, key properties of graphite change dramatically. Further, mechanical properties for metallic materials, such as those in this study, experience the largest change at irradiation levels of 0.0 dpa to $0.25 \mathrm{dpa}$.

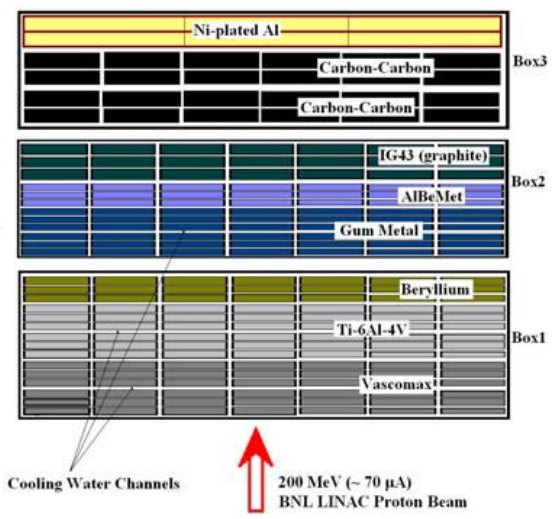

Figure 1: Specimen layout in the irradiation boxes used the BNL Phase II irradiation.

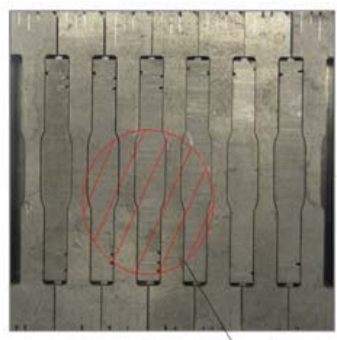

Proton Beam Footprint ( $1 \sigma$

Figure 2: Specimen arrangement in one of the layers in the irradiation assembly.

\section{POST IRRADIATION ANALYSIS}

The post-irradiation analysis in the BNL hot cell facilities, consisting of thermal expansion measurements using a sensitive dilatometer and tensile testing using a remotely operated mechanical testing machine, revealed the following regarding material response to irradiation: The $\mathrm{CC}$ composite shown in Figure 3 in its non-irradiated state exhibits negative thermal expansion. Irradiation dramatically changes that (as shown by the red trace). However, with repeated thermal cycles, the damage is annealed and slowly the material gets back to its original state. This interesting "annealing” behavior prompted the re-examination of the super-invar that was tested during phase-I and abandoned because of the dramatic effects of irradiation on its thermal expansion coefficient. What was revealed from the subsequent thermal cycling, was that super-invar also experiences annealing while reclaiming of its original thermal expansion properties. Complete recovery occurs, as seen in Figure 4, when the thermal cycling crosses the "threshold" value of $600^{\circ} \mathrm{C}$.

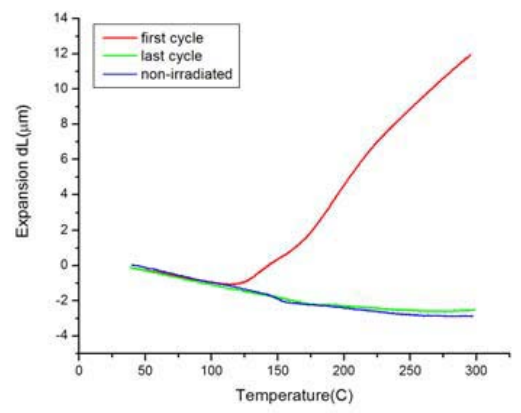

Figure 3: Thermal expansion behavior/annealing of irradiated CC composite.

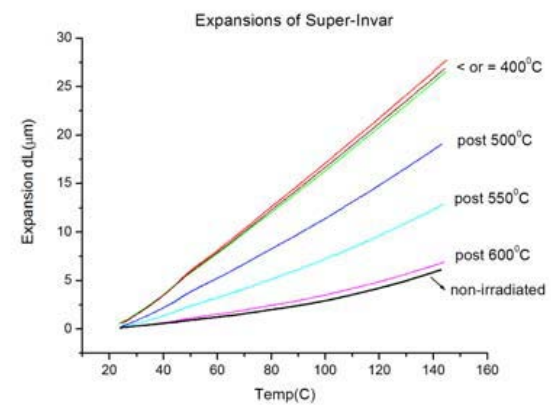

Figure 4. Thermal expansion and annealing threshold of irradiated super-invar.

Figure 5 depicts the superb properties of "gum" metal especially those of the $90 \%$ cold-worked material and in particular the high ductility that it exhibits. 

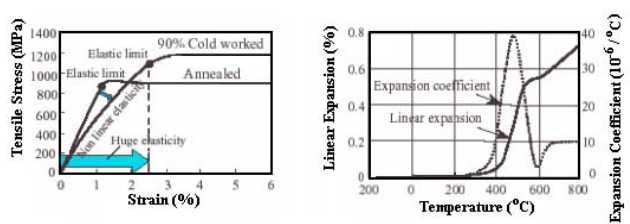

Figure 5: "Gum Metal" material characterization in its non-irradiated state

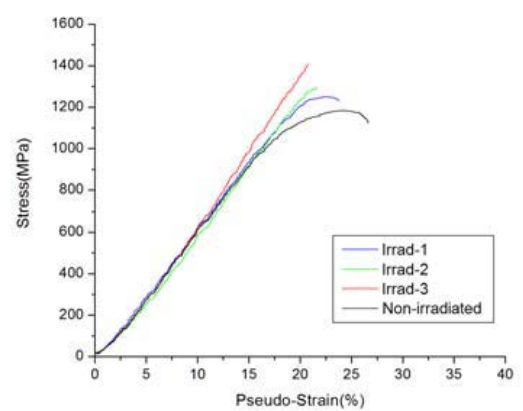

Figure 6: Irradiation effects on stress-strain relation of "gum metal".

Figure 6 clearly shows that the material ductility disappears after $0.25 \mathrm{dpa}$ of irradiation damage and it becomes totally brittle. The loss of "cold-working" in other materials has been observed in neutron irradiation studies and it is a issue that must be attended to in the next phase of the study.

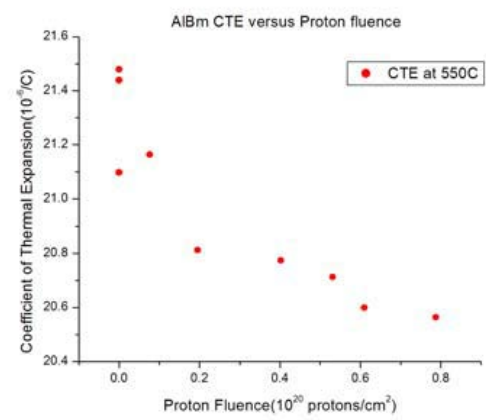

Figure 7. Irradiation effects on thermal expansion coefficient of AlBemet

As seen in Figure 8, irradiation actually reduces the thermal expansion coefficient of AlBeMet. It is the only material in the study that such a behavior was observed. In Figure 9 the stress-strain behavior of Vascomax is depicted. Interestingly, Vascomax gets stronger with irradiation but retains its ductility. This is not true, however, with the Ti-6Al-4V alloy that experiences significant ductility loss.

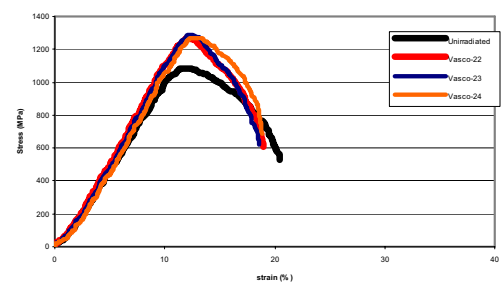

Figure 8. Vascomax stress-strain behavior

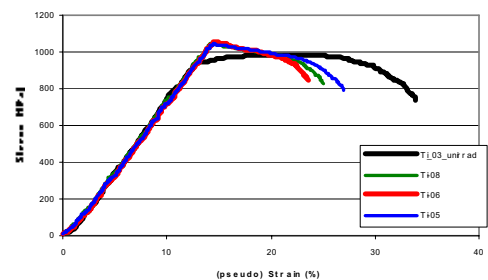

Figure 9. Ti-6Al-4v stress-strain behavior

\section{CONCLUSIVE REMARKS}

The irradiation and post-irradiation analysis of an array of materials ranging from low- $Z$ to high- $Z$, provided some interesting preliminary results of importance in the search for high power target materials. Specifically, the observation and identification of "annealing" and irradiation damage correction in some materials is a very important finding with significant consequences. Further, the loss of "cold-work" in some materials with little irradiation damage is quite troubling and needs further study. Positive surprises are the low rate of ductility loss of Vascomax and the apparent "reduction" of thermal expansion coefficient of AlBemet.

\section{REFFERENCES}

1. N. Simos, et al., "Target Material Irradiation Studies for High-Intensity Accelerator Beams," NuFact04 Proceedings, 2004

2. H.G. Kirk, et.al., "Target Studies with BNL E951 at the AGS," Proc. 2001 PAC, Chicago, Il., March 2001, p. 1535.

3. APT Materials Handbook, TPO-P00-MDD-X00001, 20001.

4. K. Nishino, "Super Multifunctional Alloy "GUM METAL".Toyota CRDL., INC. Technical News.

5. MCNPX Users Manual-Version 2.1.5, L.S. Waters, ed., LANL, Los Alamos, NM. TPOE83-GUG- X-00001. (1999) 